\section{PROTEIN GLYCOSYLATION}

\section{Chaperone mutation in Tn syndrome}

Tn syndrome is a rare autoimmune disease in which subpopulations of blood cells in all lineages carry an incompletely glycosylated membrane glycoprotein, known as the Tn antigen. This truncated antigen has the sugar $N$-acetylgalactosamine $\alpha$-linked to either a serine or threonine amino-acid residue $e^{1,2}$, whereas the correct $\mathrm{T}$ antigen has an additional terminal galactose; the defect maybe due to a malfunction of the glycosylating enzyme T-synthase ${ }^{3,4}$. Here we show that Tn syndrome is associated with a somatic mutation in Cosmc, a gene on the $\mathrm{X}$ chromosome that encodes a molecular 'chaperone' that is required for the proper folding and hence full activity of T-synthase ${ }^{5}$. The production of the autoimmune Tn antigen by a glycosyltransferase enzyme rendered defective by a disabled chaperone may have implications for other Tn-related disorders such as IgA nephropathy, a condition that can result in renal failure.

We used whole blood from two male donors with Tn syndrome (C.C. and C.L.) and from 25 healthy donors (male and female) with a total of 33 Cosmc alleles between them. T-synthase activity in whole-blood cell extracts from C.C. and C.L. was significantly lower (decreased by more than 60\%) than that in control samples. Th antigens, and Th antigens carrying additional sialic acid sugar residues, were present on erythrocytes and leukocytes from C.C. and C.L., but not on blood cells from healthy donors.

The T-synthase gene ( $T$-syn; chromosome position, 7p14-p13) contains three exons ${ }^{3}$, whereas Cosmc has a single exon of 954 base pairs (chromosome position, $\left.\mathrm{Xq}_{2} 23\right)^{5}$. To determine whether the defective T-synthase activity in C.C. and C.L. might be correlated with mutations in these genes, we sequenced Cosmc and T-syn from whole blood cells. T-syn sequences were normal for all donors, but Cosmc sequences from C.C. and C.L. were mosaic, containing both normal and mutated sequences. Cosmc from C.C. has a substitution at nucleotide $\mathbf{2 0 2}$ that gives a premature stop codon instead of an arginine residue at position 68 , and a polymorphism at nucleotide 393 that causes a conservative change from aspartate to glutamate at position 131; Cosmc from C.L is mutated at nucleotide 454 to give lysine instead of glutamate at position 152 (Fig. 1a, and see supplementary information).

We found that the mutation at nucleotide 202 occurred in 6 of 14 Cosmc clones from C.C. and that the change at nucleotide 393 was present in all 14 of his clones; C.L.'s nucleotide 454 mutation occurred in 6 of 8 clones. As Cosmc is X-linked and the two donors are male, these Cosmc sequences must be mutated in only a subset of blood cells in both. Normal Cosmc sequences from the 25 healthy donors, representing 33 alleles, were identical ${ }^{5}$. The mutation in Cosmc found in the two donors with Tn syndrome is statistically significant $(P<0.01$ in Fisher's exact test).

$\mathrm{T}$-synthase activity relies on coexpression with Cosmc $c^{5}$, so to test the effect of the Cosmc
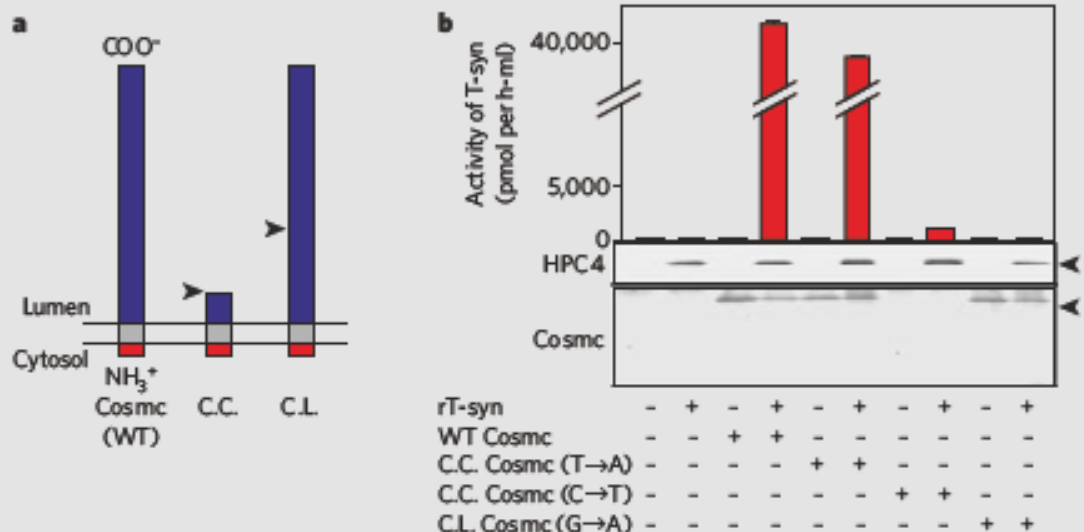

Figure 1 | Effect of Cosmc mutations on functional activity in patients with Th syndrome. a, Length comparison of newly synthesized wild-type Cosmc, which is a protein of the endoplasmic reticulum that acts as a molecular chaperone for T-synthase (T-syn), with the mutated forms in patients C.C. and C.L. (arrows indicate mutation sites). b, Effect of coexpression of wild-type (WT) or mutant forms (C.C., $+393 \mathrm{~T} \rightarrow \mathrm{A}$ or $+202 \mathrm{C} \rightarrow \mathrm{T}$; C.L., $+454 \mathrm{G} \rightarrow \mathrm{A}$, where notation numbering indicates the nucleotide mutation site and standard letter notation is used for the bases) of Cosmc on the activity of T-syn. Plasmids encoding these Cosmc variants were constructed and baculoviruses prepared in Sf- 9 cells ${ }^{5}$ (for methods, see supplementary information). Insect Hi- 5 cells were infected with baculovirusesencoding human soluble HPC4-tagged recombinant T-syn and Cosmc, as indicated. Top, T-syn activity in cell medium was measured in triplicate ( \pm s.e.m. $)$; bottom, western blots of protein in cell medium using mouse anti-HPC4 monoclonal antibody (IgG1) to detect HPC4-tagged recombinant T-syn, and blots of protein in cell extracts using chicken anti-human Cosmc polyclonal antibody (IgY) to detect Cosmc. Migration positions of T-syn $\left(M_{r}\right.$ about $\left.40 \mathrm{~K}\right)$ and $\operatorname{Cosmc}\left(M_{r}\right.$ about $\left.37 \mathrm{~K}\right)$ are indicated by arrowheads. mutations on the chaperone's function, we expressed recombinant Cosmc (wild type and mutants) together with $T$-syn in the insect cell line known as Hi-5 (Fig. 1b). As expected, coexpression of T-syn with Cosmc from C.C. that had the conservative amino-acid substitution (thymine-to-adenine polymorphism at nucleotide 393) gave normal T-synthase activity. However, coexpression with C.C.'s truncated mutant Cosmc (cytosine changed to thymine at nucleotide 202) gave less than $10 \%$ of the T-synthase activity associated with wild-type Cosmc, and no activity was detectable with C.L.'s Cosmc mutant. Expression of recombinant $T$-syn in Hi-5 cells was equivalent in all cases.

These results indicate that the specific mutations in Cosmc from patients with Tn syndrome cause it to lose its chaperone function. We confirmed by western-blot analysis that Cosmc protein was normally expressed from complementary DNA encoding wildtype Cosmc or C.C's polymorphic Cosmc. By contrast, C.C.'s truncated 68-amino-acid Cosmc was not detected, although C.L.'s mutant Cosmc, which had no chaperone activity, was detected and was normal in size.

It has been suggested that Tn syndrome is clonal and somatic ${ }^{6-8}$. Our findings indicate that a somatic mutation in Cosmc in a subpopulation of multipotential haematopoietic stem cells in patients with Tn syndrome inhibits its chaperone activity and leads to inactivation of T-synthase and the expression of the autoimmune Tn antigen on blood cells of all lineages. This discovery may provide insight into the molecular basis for other Tn-related disorders, such as IgA nephropathy ${ }^{9}$ and Henoch-Schönlein purpura ${ }^{9}$, in which somatic mutations in Cosmc in haematopoietic precursors could contribute to disease aetiology.

Tongzhong Ju, Richard D. Cummings Department of Biochemistry and Molecular Biology, and Oklahoma Center for Medical Glycabiology, University of Oklahoma Health Sciences Center, Oklahoma City, Oklahoma 73104, USA

e-mait richard-cummings@ouhsc.edu

1. Berger, E G. Blochin. Blophys. Acta 1455, 255-268(1999)

2. Cartron I.P. \& Nurden, A. T. Nature 282, 621-623 (1979).

3 Ju, T, Brewec, K, D Souza, A, Cummings, R. D.\& Canfield W.M. J.Bial. Chem. Z7, 178-186 (2002)

4. Xia, L etal J.Cell Bbl. 164, 451-459 (2004).

5. Ju, T. \& Cummings, R. D. Proc. NatI Acad Sci. USA 99, $16613-16618(2002)$

6. Cartron $\perp P_{7}$ Cartron $1, A$ Andreu G, Salmon C \& Bid, G.W. Lancet 1,856-857 (1978).

7. Felner, K M, Dinter, A. Cartron, I.P. \& Berger, E G Blachim. Blophys. Adta 1406, 115-125 (1998)

8. Moreau, R, Dausset, 1 , Bernard, I \& Moullec, J. Bull. Mem. Soc. Med. Hop. Panis 73, 569-587(1957).

9. Jullan, B. A.\& Novak, I Curr. Opin. Nephral. Hypertens 13, $171-179$ (2004).

Supplementaryinformation accompanies this communication on Nature's website. Competing interests statement: declared none. doi: $10.0138 / 4371252 a$

BRIEF COMMUNICATIONS ARISING online I www.nature.com/bca see Nature contents. 